1.07 (1.27) per individual per annum $(\mathrm{p}<0.0001)$ and was most significant in the COPD group $(\mathrm{p}=0.005)$. All patient groups indicated that HMV improved their quality of life.

Conclusion This study has demonstrated that contrary to existing evidence, the adherence to HMV was extremely good across all diseases. Remote monitoring may play a role in this increased adherence. Although HMV contributed to improvements in blood gases at 3 months, there was a universal trend for these parameters to return to baseline at 6 months in all disease states despite good adherence. Our data therefore suggests that early follow-up is essential to detect deterioration and support the need for palliation. Nevertheless HMV remains an effective treatment to reduce hospital admissions and improve quality of life, if patients are adherent to the intervention.

\section{S59 UTILITY OF AN AUTO-TITRATING PROTOCOL FOR THE SETUP OF NOCTURNAL NON-INVASIVE VENTILATION}

C Carlin, G McDowell, C Williams, A Brown, C Canavan, R Tourish. Department of Respiratory Medicine, Queen Elizabeth University Hospital, Glasgow, UK

\subsection{6/thoraxjn--2016-209333.65}

Background The prevalence of conditions requiring nocturnal breathing support is increasing. Evidence is accumulating that early delivery of optimised nocturnal non-invasive ventilation (nNIV) improves acceptability and longer term NIV usage. The AVAPS-AE (adaptive volume assured pressure support with autoEPAP) mode of the Respironics A40 ventilator offers prospects of fully auto-titrated NIV. After obtaining initial experience, we adopted use of this ventilator mode for initial nNIV titration in patients with nocturnal hypoventilation (excluding those with neuromuscular disorders), with aim of improving service efficiency and patient outcomes.

Methods Patients with nocturnal hypoventilation disorders (majority obesity-related) attend our tertiary inpatient breathing support service. In early 2014 we established a protocol for autoNIV setup which consists of first night sleep study with morning capillary blood gases (+ancillary cardiorespiratory investigations if indicated), second night on AVAPS-AE mode NIV and then transition on third night to fixed ST mode bilevel NIV based on the A40 ventilator report, supported by appropriate improvement and then stability in sleep quality, daytime symptoms, ventilator integration, overnight transcutaneous and morning blood gas measurements.

Results Between March 2014 and June 2016103 patients received AVAPS-AE mode ventilation for initial setup or re-titration of nNIV. The majority of patients tolerated auto-NIV setup protocol well, and were discharged on 'fixed' NIV using a less expensive generator with the A40 derived settings, and did not subsequently require ventilator adjustments. Auto-NIV derived settings typically indicated higher backup rates and unpredictable IPAP/EPAP requirements compared with previous experience. 21 patients continue on AVAPS-AE mode NIV long term based on high pressure support or labile ventilation requirements with suboptimal clinical parameters on fixed NIV. Mean length of stay for breathing support assessment has been reduced by $>1$ day since the adoption of auto-NIV setup protocol, and clinic follow up has also been rationalised.

Conclusions Auto-NIV setup protocol achieves significant service efficiencies. This patient cohort will continue to be studied to judge other clinical benefits, but prospective clinical trials with AVAPS-AE or similar ventilator modes for acute NIV and elective outpatient nNIV are justified.

\section{Advances in Thoracic Surgery}

\section{S60 LUNG CANCER SURGICAL SURVIVAL AND VOLUME IN ENGLAND}

${ }^{1} \mathrm{D}$ West, ${ }^{2} \mathrm{P}$ Beckett, ${ }^{3} \mathrm{~A}$ Khakwani, ${ }^{3} \mathrm{R}$ Hubbard, ${ }^{2} \mathrm{R}$ Dickinson, ${ }^{2}$ W Woolhouse. ${ }^{1}$ Society of Cardiothoracic Surgeons, London, UK; ${ }^{2}$ Royal College of Physicians, London, UK; ${ }^{3}$ University of Nottingham, Nottingham, UK

\subsection{6/thoraxjnl-2016-209333.66}

Introduction The National Lung Cancer Audit has collected data for over 10 years demonstrating gradually rising resection rates in the UK. The Clinical (formerly Consultant) Outcomes Programme (COP) is an NHS England initiative, managed by HQIP, using national audit data to publish quality measures at the level of individual consultants. The lung cancer COP focusses on activity at individual surgeon level, and on survival at unit level. The first lung cancer COP in 2014 demonstrated overall 30 and 90day survival of $97.8 \%$ and $95.5 \%$.

Methods Data submitted to the NLCA for patients having curative-intent surgery who underwent surgery in 2013 was sent to the clinical lead at each surgical unit for validation and addition of responsible surgeon GMC number, with the option to add surgical cases if they were not included in the supplied dataset. Date of death was derived by a link to the Office of National Statistics. Units reporting unadjusted survival proportions more than three standard errors outside the national mean ("alarm" level) at 30 or 90 days were identified as statistical outliers.

Results All of the 28 surgical units in England participated in the audit, submitting a total of 4892 cases. Median annual unit activity was 156 resections (IQR 99-221, range 39-481). Median annual activity for individual surgeons was 39 (IQR 20-52, range 1-152). Overall 30-day survival was $98 \%$ and 90 -day survival was $96 \%$. There were no units with statistical outliers at the alarm level at 30-days and 90-days (see Figure).

Conclusion Volume of activity varies widely by unit and individual surgeon. Survival after lung cancer surgery is very high, is improving, and is not statistically significantly different across the surgical units in England. This suggests that lung cancer teams may still be risk averse when considering surgical treatment of their patients. Reasons why patients die between 30 and 90 days is worthy of further investigation. Case-mix adjustment will be needed to allow robust comparisons between units. 

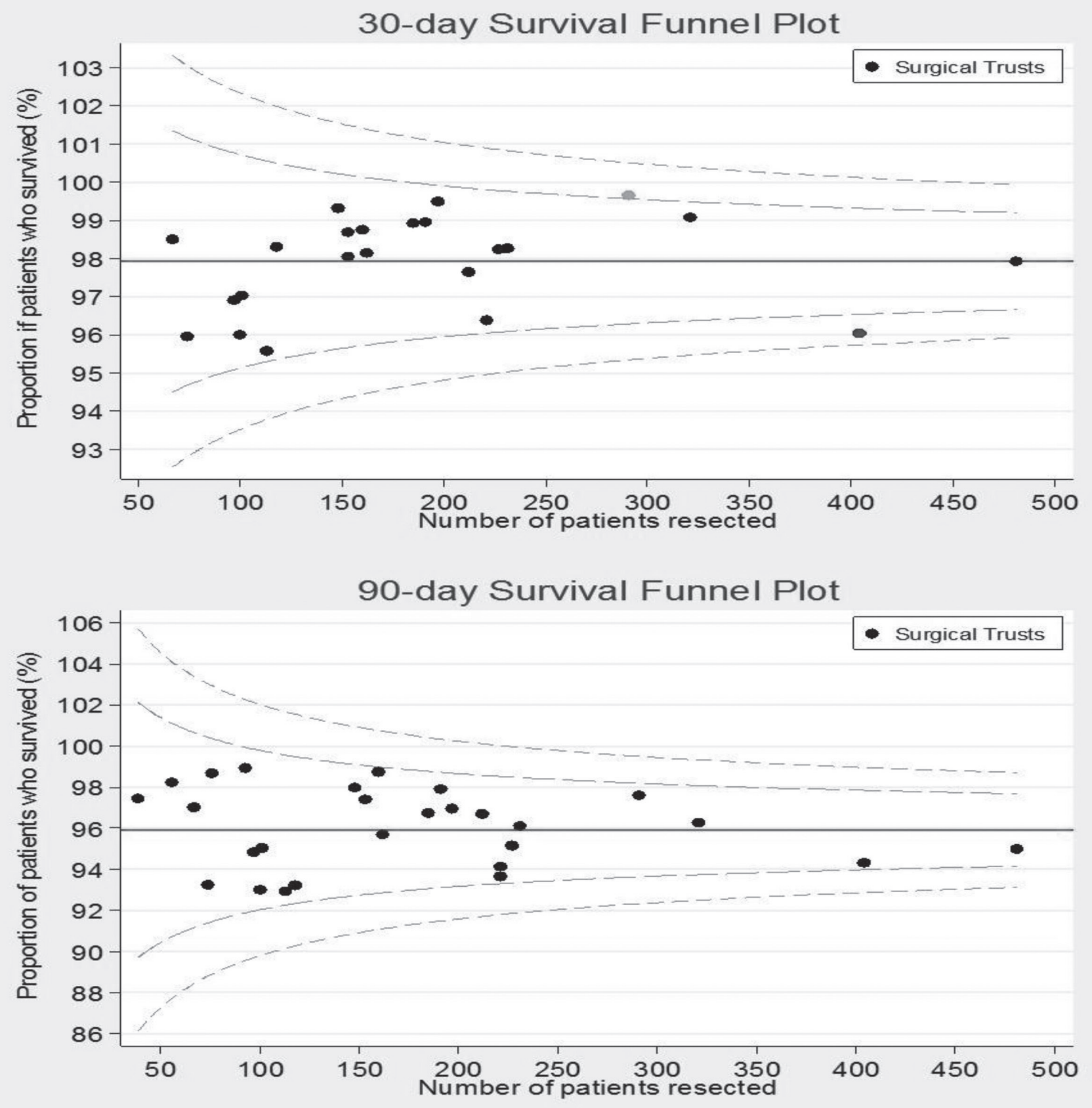

Abstract S60 Figure 130 and 90 day survival by trust

\section{S61 RISK FACTORS AND SHORT-TERM OUTCOMES OF DEVELOPING POSTOPERATIVE PULMONARY COMPLICATIONS AFTER VATS LOBECTOMY}

${ }^{1} \mathrm{P}$ Agostini, ${ }^{2}$ ST Lugg, ${ }^{1} \mathrm{~K}$ Adams, ${ }^{3} \mathrm{~T}$ Smith, ${ }^{1} \mathrm{M}$ Kalkat, ${ }^{1} \mathrm{~PB}$ Rajesh, 'RS Steyn, ${ }^{1} \mathrm{~B}$ Naidu, ${ }^{3}$ A Rushton, ${ }^{1} \mathrm{E}$ Bishay. ${ }^{1}$ Department of Thoracic Surgery, Heart of England NHS Foundation Trust, Birmingham, UK; ${ }^{2}$ Institute of Inflammation and Ageing, University of Birmingham, Birmingham, UK; ${ }^{3}$ School of Sport, Exercise and Rehabilitation Sciences, University of Birmingham, Birmingham, UK

\subsection{6/thoraxjnl-2016-209333.67}

Introduction Postoperative pulmonary complications (PPC), such as pneumonia and atelectasis are associated with poor outcomes following thoracotomy and lung resection, with risk factors identified. $^{1,2}$ Video-assisted thoracoscopic surgery (VATS) is increasingly performed, however, there are varying reports regarding the incidence of PPC with little is known about their effect on short-term outcomes or potential risk factors.

Methods A prospective observational study of consecutive patients undergoing VATS lobectomy was performed in a regional centre (2012-2016). Exclusion criteria included re-do VATS/completion lobectomy. All patients received physiotherapy assessment/intervention as necessary from postoperative day 1 (POD1). The presence of PPC was determined daily using the Melbourne Group Scale. Outcomes included hospital length of stay (LOS), intensive therapy unit (ITU) admission and hospital mortality. 